\title{
A novel GFP nude rat model to investigate tumor-stroma interactions
}

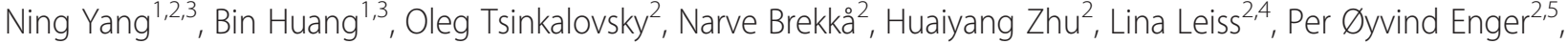 \\ Xingang Li, $i^{1,3^{*}}$ and Jian Wang ${ }^{2,3^{*}}$
}

\begin{abstract}
Backgroud: A key strategy for the study of the tumor microenvironment is to implant human tumor cells in an immunodeficient rodent strain ubiquitously expressing a fluorescent marker. Here, a novel nude rat expressing a green fluorescent protein (GFP) transgene was established and engrafted with primary human tumor tissue in order to separate tumor from stromal cell populations for subsequent molecular analysis.

Methods: SD-TG (GFP) 2BalRrrc transgenic rats were crossed with HsdHan ${ }^{\mathrm{TM}}$ : rnu/rnu Rowett nude rats to develop a GFP expressing immunocompromised rat. PCR and flow cytometry were used to follow the GFP genotype and phenotype in newborns. After three to four generations, animals were implanted with $4 \mathrm{~T} 1 \mathrm{dsRed}$ murine breast cancer cells or primary human glioblastoma (GBM) biopsies to generate xenografts for subsequent separation by fluorescence-activated cell sorting (FACS).

Results: Fluorecence microscopy and reverse transcription-PCR demonstrated that GFP, under the control of the human ubiquitin $\mathrm{C}$ promoter, was stably maintained and expressed in diverse organs over several generations. Immunophenotyping of blood samples by flow cytometry confirmed that the immunodeficient features of the parental rat strain, HsdHan ${ }^{\mathrm{TM}}$ : rnu/rnu, were retained in the GFP nude rat. Both the murine cell line and human GBM biopsies engrafted, and stromal cell populations were isolated from dissociated xenografts by FACS to > 95\% purity.

Conclusions: A GFP transgene was stably introduced into a nude rat background in which human and murine cancer cells successfully engrafted. This animal strain provides a novel in vivo system for detailed cellular and molecular characterization of tumor-stroma interactions.
\end{abstract}

Keywords: Xenograft, Tumor-stroma interaction, Glioblastoma, Breast cancer, Tumor biology

\section{Background}

The development of a human tumor is a collaboration between a mutated cell and its microenvironment. The idea that tumor growth was in part dependent on normal cell types originated over 100 years ago when Stephen Paget proposed the "seed and soil" hypothesis in 1889 [1]. Although his original studies addressed the pattern of development of metastasis in human patients, modern studies have revealed that a complex crosstalk between stromal compartments and tumor cells exists at any point during neoplastic progression [2-5].

\footnotetext{
* Correspondence: Lixg@sdu.edu.cn; Jian.Wang@biomed.uib.no 'Department of Neurosurgery, Qilu Hospital of Shandong University, Jinan, China

2Department of Biomedicine, University of Bergen, N-5009 Bergen, Norway Full list of author information is available at the end of the article
}

Stroma itself evolves during cancer development. It is composed of many different cell types, including cancerassociated fibroblasts (CAFs), endothelial cells, and diverse immune cells, as well as extracellular matrix [5]. Tumor cell signalling, survival, proliferation, and even response to chemotherapy are all affected by stroma, but its complexity makes it difficult to attribute specific functional properties to any individual cell type. Therefore, the ability to separate stromal from tumor cells to high purity is essential in order to study tumor-stroma interactions.

One ingenious way to isolate stroma has been to implant tumor cells into an immunodeficient rodent engineered to ubiquitously express a fluorescent marker protein. In the last decades, several green, red, or cyan fluorescent immunodeficient mice models, including two from our group 
[6,7], have been established where human cancer cells or biopsies successfully engrafted [8-12]. Although these models have been elegantly used to investigate the impact of stromal cell types on multiple aspects of tumor progression, the major disadvantage of xenograft models in mice is tumor size. A substantial amount of material might be required to perform, for example, both genomic and proteomic analyses. The starting cell number is especially important when xenografts are first dissociated into single cell suspensions and subsequently sorted into different cell populations by fluorescence-activated cell sorting (FACS) [6,7]. In addition, any further division of a xenograft based on the complexity of cell types present may require an even greater number of total cells at the start.

CAFs have been recognized as having an important role during the development of epithelial tumor types [13-15], and fluorescent immunodeficient mice have been an important tool in the characterization of the interaction between CAFs and tumor cells. Stroma however, has been not well described for human gliomas. Intracranial xenografts that develop from primary human glioblastomas (GBM) in nude rats recapitulate major characteristics of the disease, including angiogenesis and tumor invasion. In some cases, the xenografts express a stable invasive phenotype independent of angiogenesis over many generations in animals, and therefore, present a unique opportunity to examine molecular differences in histologically distinct stroma [16]. One of our major goals is to characterize these differences in terms of cell type and molecular profiles to aid in our understanding of the development of these tumor types. Transgenic Green fluorescent protein (GFP) rats, which are also available [17-21], are not immunodeficient and thus, are limited in their ability to generate xenograft tumors. As clinically relevant glioma models do develop in immunodeficient nude rats, in order to more effectively study tumor stroma in this model system, we wanted to develop a transgenic nude rat strain ubiquitously expressing GFP. Human GBM biopsies as well as a murine epitheial cancer cell line were implanted in GFP nude rats to generate xenograft tumors. Highly enriched stromal cell populations were isolated from the different tumor types based on fluorescence.

\section{Results}

Generation of a GFP-expressing nude rat

In order to obtain a nude rat expressing GFP, the SD-TG (GFP) 2BalRrrc transgenic rats were crossbred with $\operatorname{HsdHan}^{\mathrm{Ts}}: \mathrm{rnu} / \mathrm{rnu}$ Rowett nude rats. Newborn rats were genotyped at each generation for the presence of the GFP transgene by PCR of genomic DNA isolated from rat tails (Figure 1B). After each cross, heterozygous GFP rats and parental HsdHan ${ }^{\mathrm{rm}}$ : rnu/rnu Rowett nude rats were again crossbred to obtain heterozygous GFP nude rats and used for tumor implantation experiments after the third generation.

\section{GFP nude rats stably express GFP in diverse organs}

Evaluation of GFP expression was peformed in two ways. Firstly, fresh internal organs harvested from a heterozygous GFP nude rat were examined grossly under a UV
A
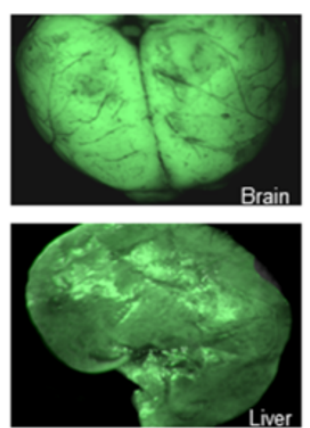

B

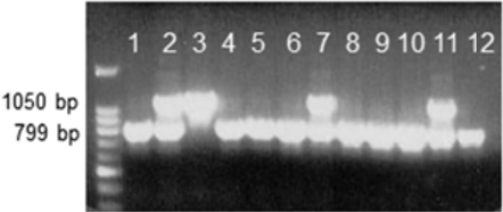

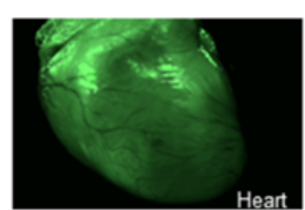
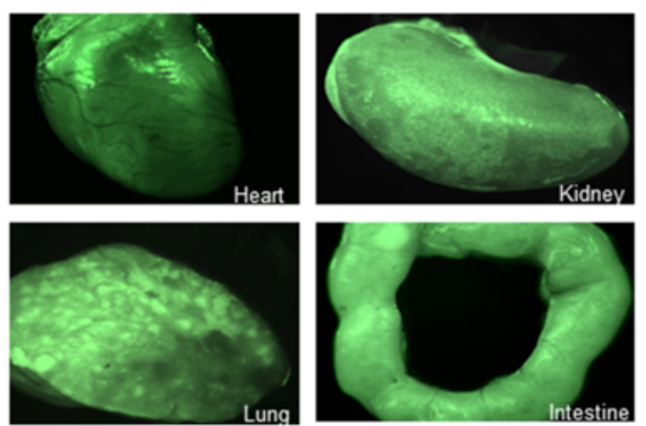

C 1400

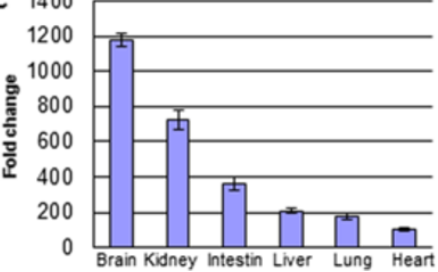

Figure 1 GFP genotyping and assessment of fluorescence expression in GFP nude rats. (A) Organs from GFP positive animals visualized under a fluorescence dissecting microscope. (B) Confirmation of the GFP genotype by PCR. Homozygous GFP rats displayed a single band at $1050 \mathrm{bp}$ (lane 3), whereas heterozygous rats exhibited bands at both 1050 and $799 \mathrm{bp}$ (lanes 2, 7, and 11). Only a single band at 799 bp was amplified from non-GFP (wild type) rats. (C) GFP expression in different organs as estimated by quantitative RT-PCR and expressed as a relative fold change compared to the organ with the lowest expression (heart). Standard error bars are indicated on the columns. 
dissecting microscope. Organs including brain, heart, kidney, intestine, lung, and liver were all highly fluorescent indicating robust GFP expression (Figure 1A). Secondly, RT-PCR was used to detect and quantify GFP expression at the mRNA level (Figure 1C). RT-PCR revealed that all organs expressed GFP at the transcriptional level. However, the expression levels differed based on tissue type; GFP was expressed at the highest levels in brain and the lowest in the heart relative to GAPDH.

\section{GFP nude rats exhibit the same immunophenotyping as} nude rats

The purpose of the cross was to integrate the GFP transgene into an immunodeficient rat background. To determine whether the homozygous GFP animals retained the immunodeficient features of the parent nude rat strain (HsdHan ${ }^{\mathrm{Tw}}$ : rnu/rnu Rowett nude rat), peripheral blood was collected from GFP nude rats and the two parental rat strains (SD-TG (GFP) 2BalRrrc transgenic and $\operatorname{HsdHan}^{\text {rw: }}$ : rnu/rnu Rowett nude rats). White blood cells were isolated, incubated with different fluorescently conjugated antibodies specific for various immune cells types ( $T, B$, and NK cells), and analyzed by flow cytometry. Both the GFP nude rats and $\operatorname{HsdHan}^{\text {tw }}: \mathrm{rnu} / \mathrm{rnu}$ Rowett nude rats lacked $\mathrm{T}$ cells (CD3) but retained B cells and NK cells (CD19 and CD161, respectively; Figure 2). These results indicated that the immune cell profile of the parental
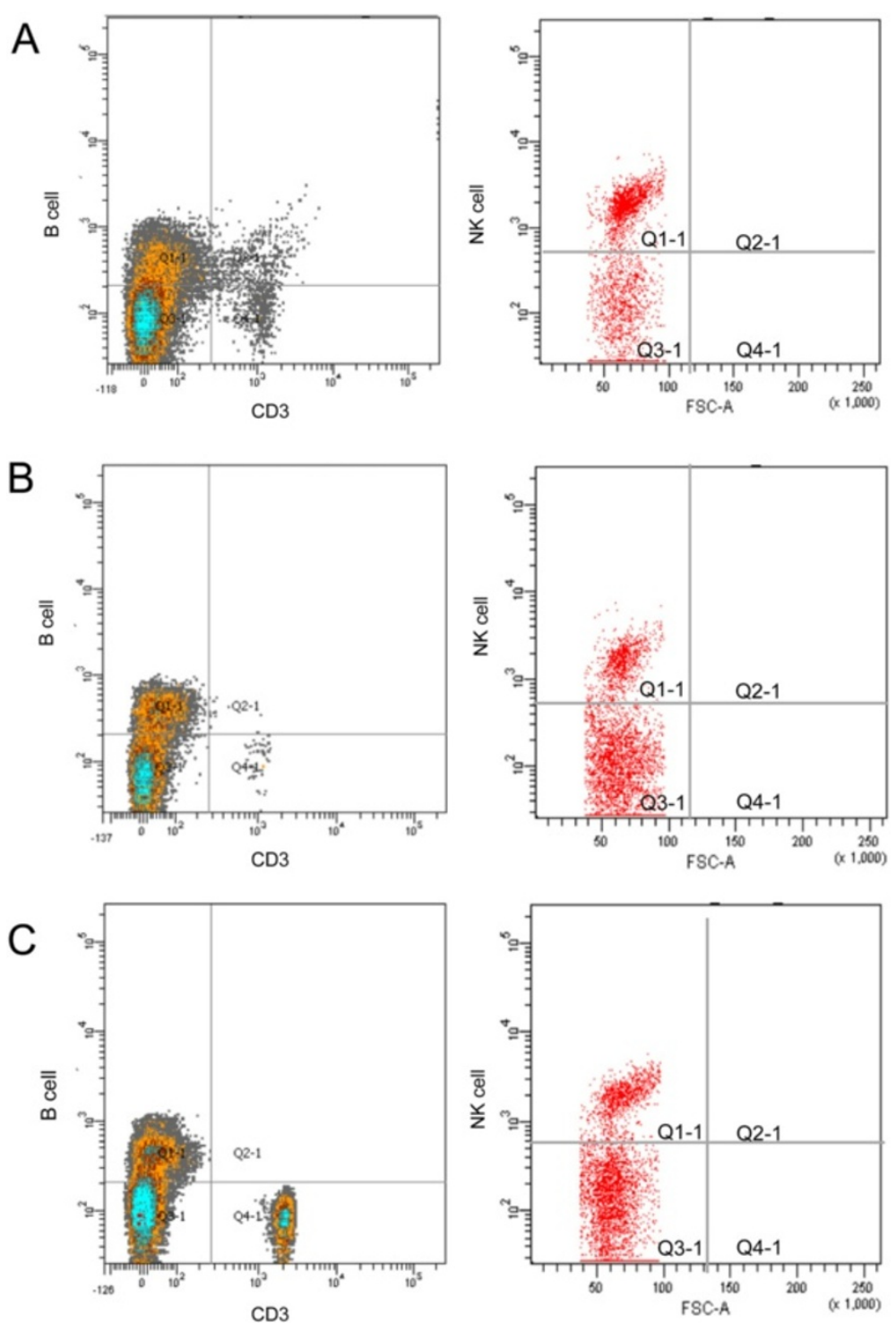

Figure 2 Immunophenotyping of GFP rat and parental strains by flow cytometry. (A) GFP nude rat; (B) nude rat; and (C) immunocompetent rat. Left panels display the distribution of B cells (PE conjugated CD19) and CD3 positive T cells (APC conjugated CD3). Right panels display the distribution of NK cells (Alexa 647 conjugated CD161). 
immunodeficient animals was preserved in the GFP crossbred nude rats.

\section{Murine breast and human GBM cell populations engraft in GFP nude rats}

One of the most important features for xenograft models is that the host animal does not reject tissue from another species. To determine whether the GFP nude rat provided a favorable environment for engraftment from other species, murine as well as human tumor cells were implanted. Orthotopic implantations were performed at two different locations, in the mammary fat pad and within the brain. For an epithelial tumor type, the murine breast cancer cell line $4 \mathrm{~T} 1$ transfected with dsRed was injected into the mammary fat pad. Tumor take was $100 \%$ at this site for $4 \mathrm{~T} 1$ cells, and tumors had reached $2 \mathrm{~cm}$ in size within 21 days.

To examine the development of gliomas in the rat brain, spheroids derived from six primary GBMs were transplanted intracranially into GFP nude rats. Animals were monitored by MRI once each week, following implantation, for tumor progression (Figure 3A, B). Tumors developed in rats for $5 / 6$ different GBMs within 6 weeks (Table 1). All animals $(n=13)$ for these five cases (P2-P6) developed tumors whereas no animal $(\mathrm{n}=2)$ developed a tumor from a single case (P1) within this timeframe. An MRI scan of a typical GBM xenograft revealed a highly aggressive tumor that was invading the contralateral hemisphere of the GFP nude rat. The lateral ventricle was also obstructed, and the tumor was growing out of the skull (Figure 3A, B). H\&E staining of the tumor revealed a somewhat circumscribed lesion with local infiltration into the surrounding normal brain tissue (Figure 3C).

High purity separation of stromal cells from tumors by FACS The models used allowed separation efficiency to be tested when both tumor and stromal cells were fluorescently labeled or when only stromal cells were labeled. Enzymatic dissociation of dsRed $4 \mathrm{~T} 1$ breast cancer xenografts from GFP nude rats (Figure 4A) generated mixed single cell suspensions as demonstrated by the presence of both red (80\%-90\%) and green fluorescent
Table 1 GBM Tumor take rate in the GFP nude rats

\begin{tabular}{ll}
\hline Patients & Take rate \\
\hline P1 & $0 / 2(0 \%)$ \\
P2 & $3 / 3(100 \%)$ \\
P3 & $2 / 2(100 \%)$ \\
P4 & $2 / 2(100 \%)$ \\
P5 & $3 / 3(100 \%)$ \\
P6 & $3 / 3(100 \%)$ \\
\hline
\end{tabular}

cells (10\%-20\%) (Figure 4B). When sorted based on fluorescence, the resultant cell populations displayed high purity ( $>95 \%$; Figure $4 \mathrm{C}, \mathrm{D}$ ).

To determine whether tumor and stromal cells could be efficiently separated based on a single fluorescent marker, fresh GBM xenografts from the GFP nude rats were enzymatically dissociated into a single cell suspension. Isolated cell populations were obtained based on GFP with high speed FACS (Figure 5A). In order to determine purity, ICC was subsequently performed on sorted cell populations with an antibody specific for the human nucleus. ICC revealed that isolated cell populations were well separated into human (red nuclei) and rat cell types (DAPI; >95\%; Figure 5B,C). The sorted cells were quantified, and the total number for cells in each compartment was compared to experiments performed with xenografts from GFP NOD/SCID mice (Table 2). In xenografts from the GFP nude rat, the total number of $\mathrm{GFP}^{+}$sorted stromal cells $\left(>10^{6}\right)$ was approximately 10 times greater than in xenografts from GFP NOD/SCID mice. GFP ${ }^{\text {neg }}$ human tumor cells comprise $80 \%$ of the xenograft in both rats and mice. The total number of GFP ${ }^{\text {neg }}$ cells sorted was approximately $5.0 \times 10^{6}$ and $1.0 \times 10^{6}$ from xenografts of GFP nude rats and GFP NOD/SCID mice, respectively. These results indicated that increased numbers of cells were routinely sorted from individual xenografts in rats based on fluorescence.

\section{Discussion}

Transgene technology allows researchers to introduce a wide range of fluorochrome genes into cells/organisms so that they can be visualized with the desired
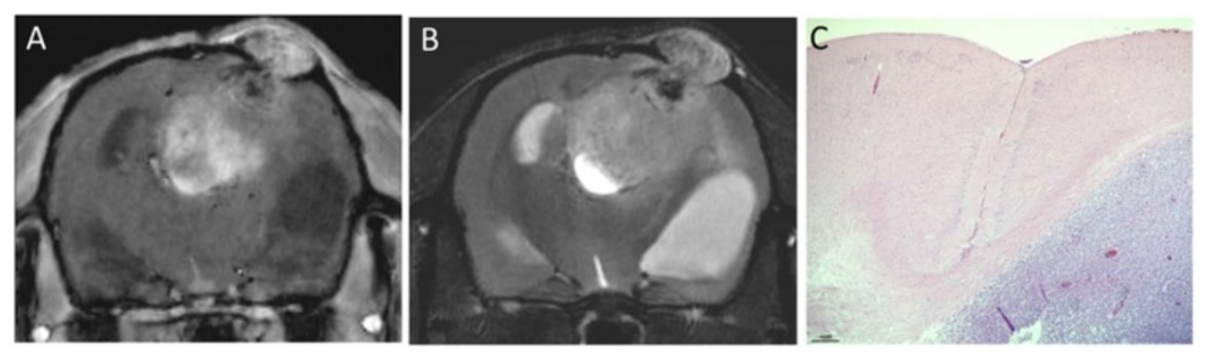

Figure 3 Engraftment of GBM biopsy in the GFP nude rat. (A) MRI scan T1 with contrast enhancement (gadolinium contrast reagent). (B) MRI scan T2 sequences reveal hydrocephalus of the animal brain. (C) H\&E staining of the corresponding tumor. 

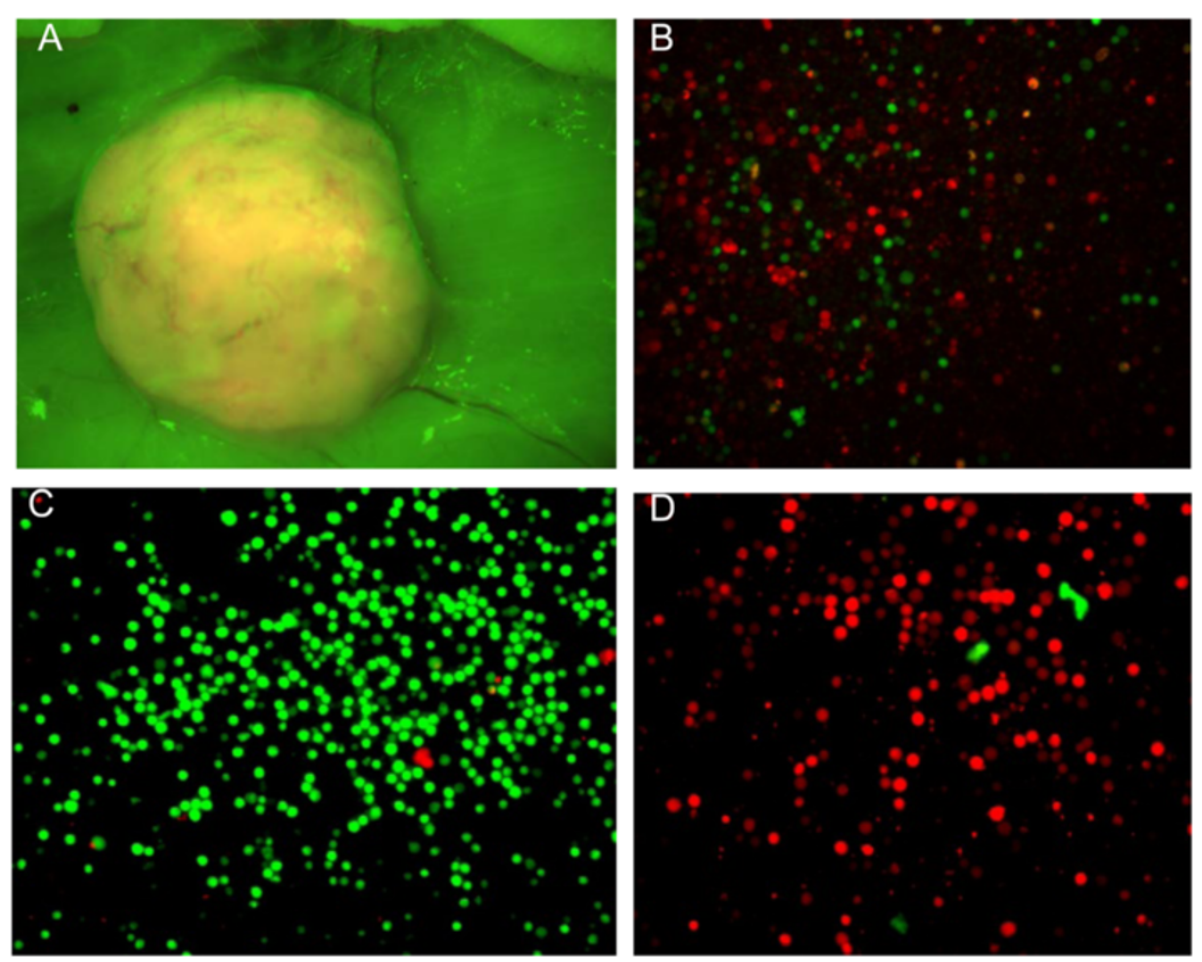

Figure 4 dsRed 4 T1 xenograft in GFP nude rat. (A) Subcutaneous 4 T1 dsRed tumor in situ after removing the skin flap of the GFP nude rat (20X magnification). (B) Cell suspension of a dissociated 4 T1 tumor displays a mixture of individual GFP expessing host cells with dsRed transfected tumor cells (20x magnification). High purity separation of (C) GFP expressing stromal cells (green) and (D) dsRed expressing 4 T1 tumor cells (red) after FACS sorting.

fluorochrome proteins in vitro and in vivo and importantly, while they are still living. Immunodeficient animals, such as nude mice, nude rats as well as SCID mice have been extensively used in cancer research because they enable xenografting of human cancer cells or biopsies, and thus generate models that more closely resemble the human tumor phenotype. Together, the two strategies produce powerful experimental tools to characterize the relationship between tumor and microenvironment. In order to begin to define the stroma for human GBM, we established a novel GFP nude rat strain by cross breeding immunocompetent GFP transgenic rats with immunodeficient nude rats. GFP was expressed in all organs examined although mRNA expression levels was dependent on the tissue type. GFP nude rats expressed the immune deficiencies of the parent nude rat, and thus, $4 \mathrm{~T} 1$ breast cancer cells as well as human GBM biopies engrafted. Finally, fluorescent stromal cells were separated from nonfluorescent or dsRed cancer cells to high purity by FACS.

In recent years, numerous fluorescent animals, such as mouse, rat, pig, and rabbit, have been established $[8,17,22,23]$. GFP transgenic rats were first introduced as a model to study organ transplantation in 2001 [17], as well as physiological aspects of a specific cell population or organ [20,24-27]. Athymic nude rats were first reported in the 1970s [28]. Since then, a plethora of experiments exploiting cancer xenografts in nude rats has been reported. The focus of our current experiments is glioma stroma, but fluorescent transgenes have been elegantly used to elaborate the evolution of stroma during tumor progression. Red fluorescent protein (RFP) positive human prostate cancer, colon cancer, breast cancer and fibrosarcoma cells have been implanted orthotopically into GFP nude mice so that tumor-host interactions could be visualized by dual-color imaging [10]. In addition, the origin of some stromal cells from the bone-marrow has been illuminated through experiments where tumor was implanted into non-transgenic SCID mice which received bone marrow transplants from GFP SCID mice [29]. Finally, multiple color proteins have been used to investigate tumor-stroma interactions in real time. Imaging was performed on live animals where cyan fluorescent protein (CFP) expressing nude mice were implanted with dualcolored human pancreatic cancer cells expressing RFP in the cytoplasm and GFP in the nucleus [11].

Even within our group, we have previously established a GFP NOD/SCID mouse strain to investigate tumorhost interactions. Orthotopic implantation of both U87 dsRed GBM and 4 T1 dsRed breast cancer cell lines generated tumors in GFP NOD/SCID mice, and stromal 

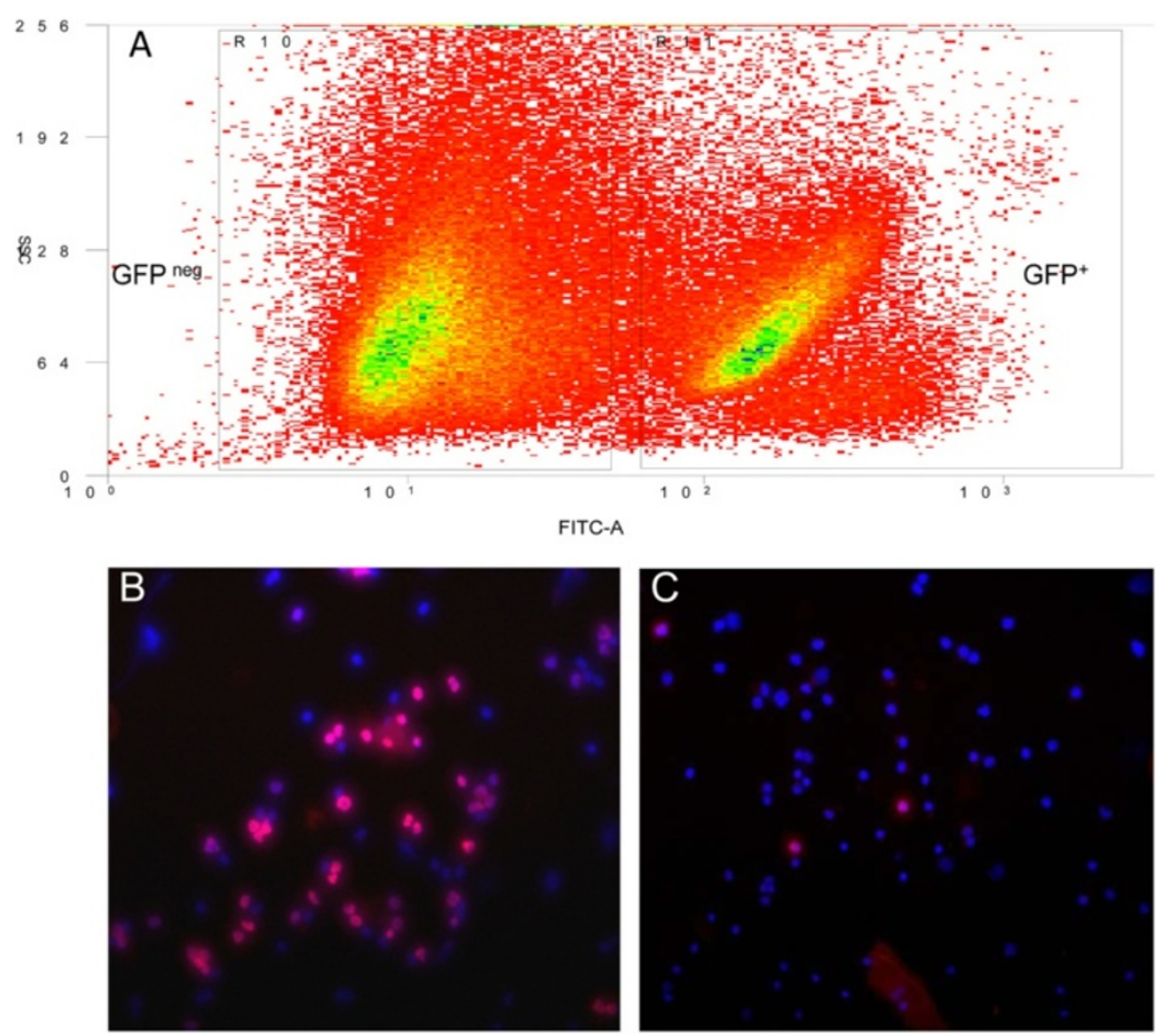

Figure 5 FACS sorting of cell populations from a GBM xenograft in the GFP nude rat. (A) Dot plot displays distinction of two cell populations, the GFP negative tumor population (GFPneg) and the GFP positive stromal population (GFP ${ }^{+}$), based on X-axis FITC fluorescence distribution. Cell populations were stained with a pan-human specific marker for human nuclear factor (HuNu) before (B) and after (C) sorting.

cells were separated by FACS to high purity [6,30]. However, homozygosity of the GFP transgene is a lethal genotype on a NOD/SCID background. Breeding is a cumbersome process as animals must be genotyped at each generation, and only heterozygous pairs used. Ultimately sufficient numbers of GFP NOD/SCID mice are slow to generate. A dsRed NOD/SCID mouse strain was also established as the fluorchrome also has favorable spectral properties at high-emission wavelengths. Homozygosity of the dsRed transgene in NOD/SCID mice is not detrimental to animals as they are completely viable and fertile [7]. For this reason, the dsRed NOD/SCID mice allow for easy breeding and maintenance of the colony.

The GFP nude rat though has several experimental advantages over fluorescent NOD/SCID mice. Firstly, when maintained under specific pathogen-free conditions, the
GFP nude rats have a maximum life span of 1.5 to 2 years or more [31] whereas NOD/SCID mice have a life span of 1 year (JAX notes). This lengthy life span of the nude rat provides sufficient time in some instances for studies regarding cancer development of more slow growing tumor types as well as to obtain survival data. In addition, NOD/SCID mice are predisposed to thymic lymphoma as well as wasting and infections which do not occur as frequently in nude rats so that more animals reach experimental endpoints. Secondly, since the immunological characteristics of the GFP nude rat are the same as the nude rat, the absence of $\mathrm{T}$ lymphocytes but the retention of $\mathrm{B}$ cell linages, the GFP nude rats can be used to study host resistance to experimental infection [31]. Finally, the size of the nude rat facilitates many aspects of in vivo experiments simply because

Table 2 Quantification of dissociated/sorted cells from a glioblastoma xenograft in GFP nude rats and GFP NOD/SCID mice

\begin{tabular}{|c|c|c|c|c|c|c|}
\hline \multirow[t]{2}{*}{ Cell compartment } & \multicolumn{3}{|c|}{ GFP nude rats } & \multicolumn{3}{|c|}{ GFP NOD/SCID mice } \\
\hline & $\mathrm{R} 1$ & R2 & R3 & M1 & M2 & M3 \\
\hline Total number of cells & $1.3 \times 10^{8}$ & $1.4 \times 10^{8}$ & $1.7 \times 10^{8}$ & $1.1 \times 10^{7}$ & $1.0 \times 10^{7}$ & $1.3 \times 10^{7}$ \\
\hline $\mathrm{GFP}^{+}$stromal cells & $1.0 \times 10^{6}$ & $1.3 \times 10^{6}$ & $1.5 \times 10^{6}$ & $2.0 \times 10^{5}$ & $2.0 \times 10^{5}$ & $2.5 \times 10^{5}$ \\
\hline GFPneg tumor cells & $5.0 \times 10^{6}$ & $5.0 \times 10^{6}$ & $5.0 \times 10^{6}$ & $1.0 \times 10^{6}$ & $1.0 \times 10^{6}$ & $1.0 \times 10^{6}$ \\
\hline
\end{tabular}


more blood and tissue material can be obtained. It is ultimately more feasible to isolate sufficient numbers of diverse cell types for molecular analyses from tumors grown in GFP nude rats. Furthermore, rats are simply much more convenient to use, particularly for intracranial surgeries, such as xenotransplantation of GBM (J. Wang, personal observation), and they are less sensitive to overdosing with anesthesia.

\section{Conclusions}

The GFP transgene has been successfully bred onto a nude rat background. Murine and human cancer cells were engrafted in these animals at a high tumor take rate, and the resulting tumors could be separated into stromal and tumor cell compartments to high purity on the basis of fluorescence. Nude rats ubiquitously expressing fluorescent markers are a unique tool for characterization of tumor stroma from diverse human cancers.

\section{Materials and methods Ethics statement}

The collection of tumor tissue from patients was approved by the Regional Ethics Committee at Haukeland University Hospital (Project number 013.09; Bergen, Norway). All patients signed informed written consent. This study was carried out in strict accordance with the recommendations in the Guide for the Care and Use of Laboratory Animals of the National Institutes of Health. The protocol was approved by the Committee on the Ethics of Animal Experiments of the University of Bergen (Permit Number: 20135415).

\section{Cell culture}

The murine breast cancer cell line 4. T1 (American Type Culture Collection, ATCC CRL-2539; Rockville, MD, USA) was transfected with a dsRed-expressing lentiviral vector (Discosoma sp. red fluorescent protein) [30]. Cells were cultured in RPMI-1640 medium (Bio-Whit-taker; Verviers, Belgium) containing fetal bovine serum (FBS;10\%), Penicillin/Streptomycin (100 units/mL), non-essential amino acids (3.2\%), plasmocin $(0.005 \mathrm{mg} / \mathrm{mL}$; InvitroGen; San Diego, CA, USA), and L-glutamine (400 $\mathrm{mol} / \mathrm{L}$; Lonza; Cologne, Germany), and were maintained in a humidified environment at $5 \% \mathrm{CO}_{2}$ and $37^{\circ} \mathrm{C}$.

\section{Tumor spheroid culture}

Six human brain tumor biopsies (GBM) were obtained from the Department of Neurosurgery, Haukeland University Hospital (Bergen, Norway). Spheroids were prepared and selected for intracerebral implantation as previously described [32]. Briefly, surgical samples were minced into $0.5 \mathrm{~mm}$ fragments and cultured in agar-coated tissue culture flasks in DMEM containing FBS (10\%). Spheroids were maintained in a humidified environment at $5 \% \mathrm{CO}_{2}$ and $37^{\circ} \mathrm{C}$, and the medium was changed once per week. After 2 weeks, ten spheroids with diameters between 200 and $300 \mu \mathrm{m}$ were selected for intracerebral implantation into rats.

\section{Animals}

SD-TG (GFP) 2BalRrrc transgenic rats (stock no. 0065, Rat Resource \& Research Center, University of Missouri; Columbia, MO, USA) harboring the GFP transgene under transcriptional control of the human ubiquitin-C promoter with a woodchuck hepatitis virus posttranscriptional regulatory element (WRE) were crossed with HsdHan $^{\text {rx: }}$ rnu/rnu Rowett nude rats (stock no. 20196, Harlan Laboratories; Horst, Netherlands) to make first generation GFP heterozygous animals [33]. Inbreeding was subsequently performed between heterozygous GFP rats and the parent $\operatorname{HsdHan}^{\mathrm{rw}}$ : rnu/rnu Rowett nude strain in order to obtain heterozygous GFP nude rats. Experimental animals could be used at the third or fourth generation after confirmation of genotype by PCR.

\section{DNA extraction and GFP genotyping by PCR}

Genomic DNA was extracted from freshly harvested tissue from rat tails according to the manufacturer's protocols (Extract-N-Amp Tissue PCR Kit; Sigma; St. Louis, MO, USA). A master mix was made which contained ExtractN-Amp PCR Reaction Mix $(10 \mu \mathrm{L})$, primers 1, 2, and 3 $(0.3 \mu \mathrm{L}$ of each at $25 \mu \mathrm{M})$, and sterile water $(5.1 \mu \mathrm{L})$. Master mix $(16 \mu \mathrm{L})$ and DNA template $(25 \mathrm{ng}$ in $4 \mu \mathrm{L})$ were added together for a final reaction volume of $20 \mu \mathrm{L}$. Cycling parameters used were the following: 1 cycle $94^{\circ} \mathrm{C}$ $3 \mathrm{~min} ; 35$ cycles $94^{\circ} \mathrm{C} 30 \mathrm{~s}, 60^{\circ} \mathrm{C} 30 \mathrm{~s}$, and $72^{\circ} \mathrm{C} 75 \mathrm{~s} ; 72^{\circ} \mathrm{C}$ for $10 \mathrm{~min}$. PCR products were analyzed on a $3 \%$ agarose gel in Tris-acetate, EDTA buffer. The wild type fragment migrated at approximately $799 \mathrm{bp}$, and the transgenic fragment at $1050 \mathrm{bp}$. Primers used for GFP genotyping of DNA were: R52 int $1 \mathrm{~F}$ : 5'-AGCAATGAATAGCC TCTCTCCT-3', R52 int 1R: 5' -CCCATATGTGCCAAGC ACTTTACC-3', U3R-0: 5' -GTCTGAAGGGATGGTTG TAGCTGT-3'.

\section{Quantitative Real Time PCR of GFP expression}

Diverse tissues from GPF nude and nude rats were harvested and immersed in RNA later solution (Ambion; Austin, TX, USA). Tissue was finely chopped and passed through a $23 \mathrm{G}$ needle five times for homogenization. RNA extraction was performed with the RNeasy Plus Mini kit (Qiagen; Hilden, Germany), according to the manufacturer's protocols. RNA was quantified on the Nanodrop ND-1000 instrument (NanoDrop products; Wilmington, DE, USA). cDNA was synthesized with the iScript Select cDNA synthesis kit (Bio-Rad; Hercules, USA) according 
to the manufacturer's protocols. Quantitative real time PCR was performed on CDNA in $\mathrm{iQ}^{\mathrm{tw}}$ SYBR Green supermix (1×; Bio-Rad, Hercules, CA, USA). cDNAs were amplified in a Bio-Rad iCycler 96 well instrument (Bio-Rad), and reactions for GFP and GAPDH were run in triplicate. Cycling parameters used were: 1 cycle $95^{\circ} \mathrm{C} 5 \mathrm{~min} ; 40$ cycles $95^{\circ} \mathrm{C} 30 \mathrm{~s}, 60^{\circ} \mathrm{C} 40 \mathrm{~s}$, and $72^{\circ} \mathrm{C} 20 \mathrm{~s} ; 1$ cycle $72^{\circ} \mathrm{C}$ $1 \mathrm{~min}$; and 1 cycle $55^{\circ} \mathrm{C} 30 \mathrm{~s}$. The following primers were used to amplify GFP and glyceraldehyde 3-phosphate dehydrogenase (GAPDH): GFP forward primer: 5'-ACG TAAACGGCCACAAGTTC-3'; GFP reverse primer: 5'AAGTCGTGCTGCTTCATGTG-3'; GAPDH forward primer: 5'-TGTGCAGTGCCAGCCTCGTC-3', GAPDH reverse primer: 5' -TGCCACTGCAAATGGCAGCC-3'.

\section{Immunophenotyping}

Peripheral blood was collected from the tails of the GFP-nude, and the parent nude and immunocompetent rats. Blood samples were treated with Easylyse buffer to lyse red blood cells (S2364, DAKO; Oslo, Norway), and the remaining cell types were pelleted and resuspended in staining buffer (2\% FBS in PBS). Cell suspensions were incubated for $30 \mathrm{~min}$ at room temperature with fluorescently conjugated antibodies against rat CD3 (eBioscience; San Diego, CA, USA), CD4, CD8, CD19 (Biolegend; San Diego, CA, USA), and isotype control. Analyses were performed on the FACS Aria II (BD Biosciences; Erembodegem, Belgium).

\section{In vivo experiments}

For implantation of breast tumor cells, dsRed-expressing 4. T1 cells were detached from monolayer with trypsin (Lonza, Cologne, Germany) and diluted in PBS. The concentration of $4 \mathrm{~T} 1$ cells was adjusted to $1 \times 10^{7}$ cells $/ \mathrm{mL}$. Rats were anesthetized with isofluran (Abbott; Oslo, Norway), and $1 \times 10^{6}$ cells were injected into the mammary fat pad. Tumor size was measured every two days by caliper. When the diameter of tumors reached $2 \mathrm{~cm}$, rats were sacrificed, and tumors were collected.

Stereotactic intracranial implantations for GBM were performed as previously described [16]. Briefly, rats were anesthetized with a mixture of $10 \mathrm{ml}$ Fentanyl $(50 \mathrm{mg} / \mathrm{mL}$; Hameln, Germany) and $1 \mathrm{~mL}$ Domitor $(1 \mathrm{mg} / \mathrm{mL}$; Orion Pharma, Finland). Rats were fixed in a stereotactic frame (Narishige Group; Tokyo, Japan), a small hole was drilled in the skull, and ten tumor spheroids were slowly injected through a wide bore Hamilton syringe (Hamilton; Reno, NV, USA) into the right frontal cortex. Rats were sacrificed when symptoms developed, and brains were removed. Primary GBM transplants were carefully dissected out from affected brains, and new biopsy spheroids were generated. Spheroids were then seeded on a plastic surface and examined ex vivo using a fluorescence microscope (Leica DMI 6000B, Leica Microsystems; Wetzlar, Germany).

\section{Fluorescence microscopy}

Fresh organs were visualized under a fluorescence dissecting microscope (Model C-DSD230; Nikon, Japan) with a UV-filter.

\section{MRI scanning}

MRI scanning was performed on anesthetized animals (1.5\% isofluoran mixed with $50 \%$ air and $\left.50 \% \mathrm{O}_{2}\right)$ in a Bruker Pharmascan $7 \mathrm{~T}$ MR scanner (Bruker Biospin MRI GmbH; Ettlingen, Germany). An axial T1 weighted MSME sequence (TR $1000 \mathrm{~ms}$, TE $8.7 \mathrm{~ms}$, slice thickness $1 \mathrm{~mm}$, FOV $3.5 \mathrm{~cm}$, matrix size $256 \times 256,20$ slices) was acquired before and after administration of subcutaneous injection of contrast agent $(1.0 \mathrm{~mL}$ of $0.5 \mathrm{mmol} / \mathrm{mL}$ Omniscan; Nycomed Amersham, Oslo, Norway). An axial T2 weighted RARE sequence was also acquired (TR $4200 \mathrm{~ms}$, TE $36 \mathrm{~ms}$, slice thickness $1 \mathrm{~mm}$, FOV $3.5 \mathrm{~cm}$, and matrix size $256 \times 256,20$ slices).

\section{Fluorescence-activated cell sorting}

4 T1 breast tumors were dissociated into single cell suspensions as previously described [30]. For GBM xenografts, fresh tissue was finely minced and dissociated into a single cell suspension with the Neural Tissue Dissociation kit (Miltenyi Biotec; Bergisch Gladbach, Germany) according to the manufacturer's protocols. The mixed cell suspensions were subsequently sorted into isolated tumor and stromal cell populations with the FACS Aria II (Becton Dickinson; Erembodegem, Belgium).

\section{Histological analysis and immunostaining}

Rat brains were fixed in $4 \%$ formaldehyde immediately following sacrifice. Tissues were embedded in paraffin, and $5 \mu \mathrm{m}$ sections were prepared and stained with Hematoxylin and eosin $(\mathrm{H} \& \mathrm{E})$ for histological analysis.

Immunocytochemistry (ICC) was performed on isolated cell populations following FACS. Mouse anti-HuNu (1:100, Millipore, MA, USA) and TXRD-conjugated goat antimouse (1:100; Southern Biotech; AL, USA) were used to distinguish human tumor cells from rat host cells. Cells were then mounted in Vectashield mounting medium with DAPI for identification of all nuclei present (Vector Laboratories; Burlingame, CA, USA). Immunocytochemistry was visualized under confocal microscopy (Leica TCS SP5; Leica Microsystems Wetzlar, Germany).

\section{Abbreviations}

GFP: Green fluorescent protein; GBM: Glioblastoma; FACS: Fluorescence-activated cell sorting; CAF: Cancer-associated fibroblasts; H\&E: Hematoxylin and eosin; ICC: Immunocytochemistry; RFP: Red fluorescent protein; CFP: Cyan fluorescent protein. 


\section{Competing interests}

The authors declare that they have no competing interests.

\section{Authors' contributions}

$N Y, X G L, P \varnothing E$ and JW were responsible for conceptualizing the study. NY, BH, OT, NB, HYZ and LL performed experiments and analyzed data. NY, PØE and JW drafted the manuscript. $P \varnothing E$ and JW critically reviewed and revised the manuscript. All the authors read and approved the manuscript.

\section{Acknowledgements}

We thank Tove Johansen, Bodil Hansen and Halala Saed for their technical assistance. The study was supported by the Bergen Medical Research Foundation (JW, 2010) and the National Natural Science Foundation of China (Grant number: 81172404, 81372720); XGL and JW were supported by the Special Fund for Taishan Scholarship Project; NY was supported by the China Scholarship Council (Grant number CSC201206220069); BH was supported by the Shandong Provincial Foundation for Distinguished Young Scholars (BS2012YY016).

\section{Author details}

${ }^{1}$ Department of Neurosurgery, Qilu Hospital of Shandong University, Jinan, China. ${ }^{2}$ Department of Biomedicine, University of Bergen, N-5009 Bergen, Norway. 'Brain Science Research Institute, Shandong University, Jinan, China. ${ }^{4}$ Neuro Clinic, Haukeland University Hospital, Bergen, Norway. ${ }^{5}$ Department of Neurosurgery, Haukeland University Hospital, Bergen, Norway.

Received: 10 September 2014 Accepted: 11 December 2014 Published online: 21 December 2014

\section{References}

1. Stwphen P: The distribution of secondary growths in cancer of the breast. Lancet 1889, 133(3421):571-573.

2. Axelrod R, Axelrod DE, Pienta KJ: Evolution of cooperation among tumor cells. Proc Natl Acad Sci U S A 2006, 103(36):13474-13479.

3. Kalluri R, Zeisberg M: Fibroblasts in cancer. Nat Rev Cancer 2006, 6(5):392-401

4. Langley RR, Fidler IJ: Tumor cell-organ microenvironment interactions in the pathogenesis of cancer metastasis. Endocr Rev 2007, 28(3):297-321.

5. Pietras K, Ostman A: Hallmarks of cancer: interactions with the tumor stroma. Exp Cell Res 2010, 316(8):1324-1331.

6. Niclou SP, Danzeisen C, Eikesdal HP, Wiig H, Brons NH, Poli AM, Svendsen A, Torsvik A, Enger $P \varnothing$, Terzis JA, Bjerkvig R: A novel eGFP-expressing immunodeficient mouse model to study tumor-host interactions. FASEB J 2008, 22(9):3120-3128.

7. Jacobsen HK, Sleire L, Wang J, Netland IA, Mutlu E, Forde H, Pedersen PH, Gullberg D, Enger PO: Establishment of a novel dsRed NOD/Scid mouse strain to investigate the host and tumor cell compartments. Cancer Invest 2013, 31(4):221-230.

8. Okabe M, Ikawa M, Kominami K, Nakanishi T, Nishimune Y: 'Green mice' as a source of ubiquitous green cells. FEBS Lett 1997, 407(3):313-319.

9. Yang M, Li L, Jiang P, Moossa AR, Penman S, Hoffman RM: Dual-color fluorescence imaging distinguishes tumor cells from induced host angiogenic vessels and stromal cells. Proc Natl Acad Sci U S A 2003, 100(24):14259-14262.

10. Yang M, Reynoso J, Jiang P, Li L, Moossa AR, Hoffman RM: Transgenic nude mouse with ubiquitous green fluorescent protein expression as a host for human tumors. Cancer Res 2004, 64(23):8651-8656.

11. Tran Cao HS, Reynoso J, Yang M, Kimura H, Kaushal S, Snyder CS, Hoffman RM, Bouvet M: Development of the transgenic cyan fluorescent protein (CFP)expressing nude mouse for "Technicolor" cancer imaging. J Cell Biochem 2009, 107(2):328-334.

12. Yang M, Reynoso J, Bouvet M, Hoffman RM: A transgenic red fluorescent protein-expressing nude mouse for color-coded imaging of the tumor microenvironment. J Cell Biochem 2009, 106(2):279-284.

13. Gaggioli C, Hooper S, Hidalgo-Carcedo C, Grosse R, Marshall JF, Harrington K, Sahai E: Fibroblast-led collective invasion of carcinoma cells with differing roles for RhoGTPases in leading and following cells. Nat Cell Biol 2007, 9(12):1392-1400.

14. Levental KR, Yu H, Kass L, Lakins JN, Egeblad M, Erler JT, Fong SF, Csiszar K, Giaccia A, Weninger W, Yamauchi M, Gasser DL, Weaver VM: Matrix crosslinking forces tumor progression by enhancing integrin signaling. Cell 2009, 139(5):891-906.
15. Ostman A, Augsten M: Cancer-associated fibroblasts and tumor growth-bystanders turning into key players. Curr Opin Genet Dev 2009, 19(1):67-73

16. Wang J, Miletic $H$, Sakariassen $P \varnothing$, Huszthy PC, Jacobsen H, Brekkå N, Li X, Zhao P, Mørk S, Chekenya M, Bjerkvig R, Enger $P \varnothing$ : A reproducible brain tumour model established from human glioblastoma biopsies. BMC Cancer 2009, 9:465.

17. Hakamata Y, Tahara K, Uchida H, Sakuma Y, Nakamura M, Kume A, Murakami T, Takahashi M, Takahashi R, Hirabayashi M, Ueda M, Miyoshi I, Kasai N, Kobayashi E: Green fluorescent protein-transgenic rat: a tool for organ transplantation research. Biochem Biophys Res Commun 2001, 286(4):779-785.

18. Mothe AJ, Kulbatski I, van Bendegem RL, Lee L, Kobayashi E, Keating A, Tator $\mathrm{CH}$ : Analysis of green fluorescent protein expression in transgenic rats for tracking transplanted neural stem/progenitor cells. $J$ Histochem Cytochem 2005, 53(10):1215-1226.

19. Francis JS, Olariu A, Kobayashi E, Leone P: GFP-transgenic Lewis rats as a cell source for oligodendrocyte replacement. Exp Neurol 2007, 205(1):177-189.

20. Moloney TC, Dockery P, Windebank AJ, Barry FP, Howard L, Dowd E: Survival and immunogenicity of mesenchymal stem cells from the green fluorescent protein transgenic rat in the adult rat brain. Neurorehabil Neural Repair 2010, 24(7):645-656.

21. Kemp SW, Phua PD, Stanoulis KN, Wood MD, Liu EH, Gordon T, Borschel GH: Functional recovery following peripheral nerve injury in the transgenic Thy1-GFP rat. J Peripher Nerv Syst 2013, 18(3):220-231.

22. Naruse $K$, Ishikawa $H$, Kawano HO, Ueda H, Kurome M, Miyazaki K, Endo M, Sawasaki T, Nagashima H, Makuuchi M: Production of a transgenic pig expressing human albumin and enhanced green fluorescent protein. J Reprod Dev 2005, 51(4):539-546.

23. Wang HJ, Lin AX, Zhang ZC, Chen YF: Expression of porcine growth hormone gene in transgenic rabbits as reported by green fluorescent protein. Anim Biotechnol 2001, 12(2):101-110.

24. Itakura E, Odaira K, Yokoyama K, Osuna M, Hara T, Inoue K: Generation of transgenic rats expressing green fluorescent protein in S-100betaproducing pituitary folliculo-stellate cells and brain astrocytes. Endocrinology 2007, 148(4):1518-1523.

25. Horiguchi K, Fujiwara K, Kouki T, Kikuchi M, Yashiro T: Immunohistochemistry of connexin 43 throughout anterior pituitary gland in a transgenic rat with green fluorescent protein-expressing folliculo-stellate cells. Anat Sci Int 2008, 83(4):256-260.

26. Popova E, Rentzsch B, Bader M, Krivokharchenko A: Generation and characterization of a GFP transgenic rat line for embryological research. Transgenic Res 2008, 17(5):955-963.

27. Gay VL, Hemond PJ, Schmidt D, O'Boyle MP, Hemond Z, Best J, O'Farrell L, Suter KJ: Hormone secretion in transgenic rats and electrophysiological activity in their gonadotropin releasing-hormone neurons. Am J Physiol Endocrinol Metab 2012, 303(2):E243-E252.

28. Festing MF, May D, Connors TA, Lovell D, Sparrow S: An athymic nude mutation in the rat. Nature 1978, 274(5669):365-366.

29. Udagawa T, Puder M, Wood M, Schaefer BC, D'Amato RJ: Analysis of tumorassociated stromal cells using SCID GFP transgenic mice: contribution of local and bone marrow-derived host cells. FASEB J 2006, 20(1):95-102.

30. Moen I, Jevne C, Wang J, Kalland KH, Chekenya M, Akslen LA, Sleire L, Enger $P \varnothing$, Reed RK, Øyan AM, Stuhr LE: Gene expression in tumor cells and stroma in dsRed $4 \mathrm{~T} 1$ tumors in eGFP-expressing mice with and without enhanced oxygenation. BMC Cancer 2012, 12:21.

31. Schuurman HJ, Hougen HP, van Loveren H: The rnu (Rowett Nude) and rnuN (nznu, New Zealand Nude) Rat: An Update. ILAR J 1992, 34(1-2):3-12.

32. Bjerkvig R, Tonnesen A, Laerum OD, Backlund EO: Multicellular tumor spheroids from human gliomas maintained in organ culture. $J$ Neurosurg 1990, 72(3):463-475.

33. Lois C, Hong EJ, Pease S, Brown EJ, Baltimore D: Germline transmission and tissue-specific expression of transgenes delivered by lentiviral vectors. Science 2002, 295(5556):868-872. 